\title{
Los caminos a la inter y transdisciplina de la Universidad Iberoamericana
}

\section{Pathways to Inter and Transdisciplinarity at Universidad Iberoamericana}

\author{
Celia Arteaga Conde \\ Universidad Iberoamericana Ciudad de México, México \\ celia.arteaga@ibero.mx \\ José Alberto Lara Pulido \\ Universidad Iberoamericana Ciudad de México, México \\ jose.lara@ibero.mx \\ ORCID: https://orcid.org/0000-0003-1484-8451 \\ José Alberto Gallardo Cruz \\ Universidad Iberoamericana Ciudad de México, México \\ jose.gallardo@ibero.mx \\ ORCID: https://orcid.org/0000-0002-0509-7003 \\ Carmina Isabel Jiménez Quiroga \\ Universidad Iberoamericana Ciudad de México, México \\ carmina.jimenez@ibero.mx \\ ORCID: https://orcid.org/0000-0002-9989-0036

\section{Adriana Carolina Flores Díaz} \\ Universidad Iberoamericana Ciudad de México, México \\ adriana.flores@ibero.mx \\ ORCID: https://orcid.org/0000-0003-1297-7380

\section{Raúl Gutiérrez Patiño} \\ Universidad Iberoamericana Ciudad de México, México \\ raul.gutierrez@ibero.mx \\ ORCID: https://orcid.org/0000-0002-8305-0825

\section{Juan Manuel Núñez} \\ Universidad Iberoamericana Ciudad de México, México \\ juan.nunez@ibero.mx \\ ORCID: https://orcid.org/0000-0002-9835-0599 \\ https://doi.org/10.48102/didac.2021..78_JUL-DIC.81
}




\title{
RESUMEN
}

Este trabajo pretende abrir un diálogo sobre las experiencias de inter y transdisciplina al interior de los programas formativos de la Universidad Iberoamericana (Ibero). El proceso de construcción se realizó a partir de una revisión teórica y conceptual, así como de la aplicación de herramientas mixtas que recogen las experiencias y perspectivas de los grupos académicos a través del diálogo, entrevistas semiestructuradas y de encuestas destinadas a los directores de las divisiones y a los directores y coordinadores de los departamentos y centros de la universidad.

Los resultados obtenidos muestran que una tercera parte de los programas que ofrece la universidad se encaminan principalmente hacia la inter y transdisciplina mediante planes de estudio, prácticas profesionales y servicio social. Lo anterior permite iniciar un diálogo y plantear una serie de reflexiones que se orienten hacia la construcción de una comunidad de aprendizaje.

Nuestra propuesta, desde el Centro Transdisciplinar Universitario para la Sustentabilidad (Centrus), es construir, de manera conjunta, una agenda y una hoja de ruta, para quienes queremos tomar este rumbo, en el que se incluyan a todos los actores involucrados dentro y fuera de la academia; que se trabaje de forma colaborativa en proyectos viables, y se otorgue un acompańamiento puntual y continuo a la difusión y medición de los resultados.

Palabras clave: Aprendizaje inter y transdisciplinar; innovación educativa; programas docentes; comunidad de aprendizaje.

\begin{abstract}
The following study aims to start a dialogue about the inter and transdisciplinary experiences within the Universidad Iberoamericana's academic offer. We carried out a theoretical and conceptual review, as well as the use of mixed research tools to collect experiences and perspectives of its academic groups through conversation and by conducting semi-structured interviews and surveys to Division Directors and Coordinators of the various University Departments and Centers.

The results show that one-third of the programs offered by the University is perceived within the path of Inter and Transdisciplinary approach due mainly to their study programmes, professional practices, and social service. This is what enables us to start an exchange of ideas and propose a series of final observations aimed towards the construction of a learning community.

The University Transdisciplinary Center for Sustainability of Universidad Iberoamericana, (Centrus, for its acronym in Spanish), proposes to jointly build an agenda and roadmap for those of us who want to choose this path, which should include every player involved within the Academy and out of it; where collaborative work should be done in viable projects and accurate and continuous support should be granted to dissemination and measurement of results.
\end{abstract}

Keywords: Educational Innovation; Inter and Ttransdisciplinary Learning; Learning Community; Teaching Programs.

Fecha de recepción: 09/04/2021

Fecha de aceptación: 05/05/2021

Introducción: nuestro punto de partida

En la actualidad, se sabe que la interdisciplina y transdisciplina son resultado de iniciativas colaborativas que buscan atender un problema de investigación, o bien, generar conocimiento en torno a éste. En este sentido, la transdisciplina constituirá un enfoquemétodo-práctica que promoverá la contribución activa de personas más allá de la academia (Mauser et al., 2013), además de saberes distintos a los provenientes de la ciencia occidental. En ambos casos, 
estas iniciativas involucran tareas ligadas a procesos de investigación, enseñanza y aprendizaje.

Además del aprendizaje de contenidos temáticos referentes al problema analizado, los equipos que participan en las iniciativas interdisciplinarias y transdisciplinarias tienen el potencial para desarrollar competencias que coadyuven a la construcción de esos espacios colaborativos. Así pues, la academia enfrenta el reto de recuperar los aprendizajes inter y transdisciplinarios para construir una pedagogía a partir de esos "nuevos saberes y competencias" derivados de las experiencias innovadoras. ¿Qué caminos hay que seguir para incorporar a los grupos de docentes y al alumnado en estas iniciativas? ¿Cómo construir nuevas configuraciones institucionales que sean el soporte del quehacer inter y transdisciplinar?

Estos procesos, particularmente los ligados al aprendizaje, permiten la formación del alumnado para coadyuvarlos a generar un pensamiento crítico y reflexivo, es decir, como personas que trabajan en equipo y de manera colaborativa (Thompson, en Carrizo et al., 2004). En la educación superior, la enseñanza y el aprendizaje posibilitan que los investigadores, el profesorado y el alumnado se conviertan en "agentes de cambio" (Scholz \& Steiner, 2015a) para hacer frente a problemas complejos, lo cual representa varios desafíos que acarrea la tradición disciplinar que caracteriza a las universidades (Carrizo et al., 2004). Asimismo, resulta indispensable desarrollar estructuras horizontales, flexibles, críticas y autocríticas que impulsen la colaboración y creación de metodologías y epistemologías que generen conocimientos que respondan a las necesidades de la propia universidad y del medio social, cultural, ambiental, económico y político (Hernández, 2016) que, a su vez, ayuden a establecer una postura ética donde los participantes de las iniciativas colaborativas converjan en un espacio seguro de interacción. $\mathrm{Al}$ asumir este reto, la Ibero debe revisar su oferta actual, así como conocer la perspectiva de los docentes frente a este aspecto. Aunado a esto, se deben tomar en cuenta los alcances de la legislación en materia de educación a partir de su relación con las universidades y la creación de programas de estudio con base en enfoques pedagógicos que favorezcan el ejercicio de la inter y transdisciplina como sinónimo de efectividad para la formación integral de los profesionistas.

El objetivo central de este trabajo, por tanto, fue realizar un diagnóstico sobre cómo son percibidos los programas educativos vigentes de la Ibero (planes suJ, para el caso de licenciatura) en función de los espacios que ofrecen para incorporar la visión y práctica inter y transdisciplinarias. Para esto, se utilizaron herramientas mixtas que recogen las experiencias y perspectivas de los grupos académicos. Este ejercicio busca contribuir a la reflexión acerca de las formas y los caminos que va tomando la incorporación de la inter y transdisciplina en la función de la docencia, investigación y vinculación (Universidad Iberoamericana, 2018). Además, se pretende recuperar lo que ha significado para los grupos académicos de la universidad la creación y gestión de estos programas, así como saber hacia dónde van y qué alcances se esperan obtener.

\section{Las vertientes de la inter y transdisciplina y sus} caminos

La especialización de la ciencia en múltiples y diversas disciplinas ha fomentado que los conocimientos se generen de manera fragmentada, lo que ha favorecido así la separación del quehacer científico (Vilar, 1997; Mauser et al., 2013). Sin duda, esto ha originado un sinfín de avances científicos y tecnológicos, aunque también presenta ciertas limitaciones (Kuhn, 2012).

El paradigma de la complejidad representa una alternativa a la forma disciplinar, ya que éste intenta vincular y distinguir, es decir, es un ir y venir entre certidumbres e incertidumbres, entre lo elemental y lo general, y entre lo separable y lo inseparable (Morin, 1996). Asimismo, propone la articulación y organización de los conocimientos para "volver a conocer de otra manera los problemas de la realidad" (Flores \& Rubio, 2019, p. 11). La interdisciplina y transdisciplina favorecen la construcción colectiva del conocimiento no solamente en términos teóricos, sino como una "exigencia práctica" (Vilar, 1997, p. 94), pues la comprensión de la rea-

90 - La caminos a la inter y transdisciplina de la Universidad Iberoamericana 
lidad necesita la conexión entre saberes ( $\mathrm{Scholz} \mathrm{\&}$ Steiner, 2015a; Kelly et al., 2016).

La interdisciplina no niega, minimiza o reduce los campos disciplinarios que están involucrados, sino que los asocia y magnifica su alcance (Carrizo et al., 2004). Plantea una integración científica que, por medio del diálogo y construcción colectivos, contrasta y supera los límites de las disciplinas (Mauser et al., 2013). Un ejemplo es "la diferencia entre enfoques cualitativos y cuantitativos o entre enfoques analíticos e interpretativos que unen disciplinas de las humanidades y las ciencias naturales" (Kelly et al., 2016, p. 6).

Por su parte, los enfoques transdisciplinarios proponen la colaboración entre personas dedicadas a la investigación con participantes que provienen de otros "campos de experiencia" (Norgaard, 1999), como el gobierno, la industria y la sociedad civil, en el uso y la construcción de diferentes epistemologías y metodologías para resolver problemas complejos y socialmente relevantes (Carrizo et al., 2004; Mauser et al., 2013; Kelly et al., 2016; Scholz \& Steiner, 2015a; Scholz \& Steiner, 2015b). En otras palabras, "es la ciencia y el arte de descubrir puentes entre diferentes objetos y áreas de conocimiento" (Thompson, en Carrizo et al., 2004, p. 32).

En algunas propuestas, el proceso de investigación transdisciplinario implica codiseño, coproducción y coevaluación del conocimiento y del proceso colaborativo (Mauser et al., 2013; Scholz \& Steiner, 2015a; Wanner et al., 2018), donde es importante recuperar el aprendizaje que nutre tanto al propio conocimiento como a la práctica. El "valor agregado" de la transdisciplina consiste en emplear diferentes formas epistémicas, múltiples metodologías, diversos saberes y la construcción constante de lenguajes y objetivos comunes (Scholz \& Steiner, 2015a; Scholz \& Steiner, 2015b). Es un giro en la idea de hacer ciencia "para la sociedad" en hacer ciencia "con la sociedad" para atender, a través del consenso, problemas sociales complejos y relevantes (Scholz \& Steiner, 2015b). La disposición a participar en esta práctica colaborativa representa uno de los puntos de anclaje de estas experiencias.

\section{Las experiencias de los caminantes}

Consideramos que la enseñanza y el aprendizaje de la inter y transdisciplina han dado resultados fructíferos para el quehacer universitario, por lo cual se expresan en diversos esquemas de enseñanza, métodos y programas (para ampliar estas reflexiones sugerimos revisar Fam et al., 2017). En este artículo mostramos dos de esas experiencias de quienes hemos llamado "caminantes" hacia la educación inter y transdisciplinar.

El Programa Glocal (The glocal curriculum) (John et al., 2017) es resultado del experimento denominado Aula Global, que buscó atender los desafíos institucionales rumbo a la sustentabilidad por parte de las universidades Leuphana y Estatal, ubicadas en Baja Sajonia y Arizona, respectivamente. Para atender tales desafíos, este grupo propuso como principios rectores de los planes de estudio "los contextos globales, los sistemas complejos, los problemas del mundo real, la sostenibilidad y una sólida base disciplinaria en el contexto de los enfoques inter y transdisciplinar" (John et al., 2017, p. 15). El Aula Global adoptó el modelo de trabajo

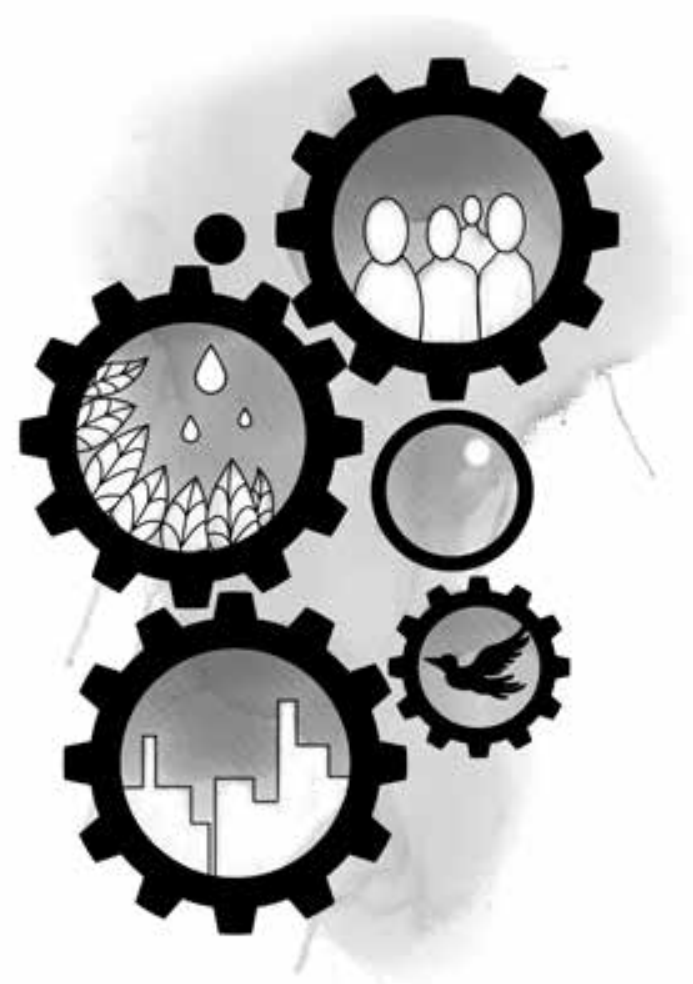


"glocal" en virtud de considerar ambas escalas para atender los retos de la sustentabilidad; se realizó en medios digitales y ha demostrado que "es posible aplicar innovaciones curriculares centradas en los problemas del mundo real, el pensamiento complejo y la naturaleza profundamente contextual del conocimiento en el mundo" (John et al., 2017, p. 15). Este grupo de investigación reporta que para esta experiencia transnacional fue crucial desarrollar competencias sociales y comunicativas en ámbitos interculturales, comúnmente consideradas dentro de los resultados deseables, aunque poco incorporadas en los programas académicos tradicionales. En su operacionalización y gestión, el Programa Glocal distingue a) la concepción-visualización de los planes de estudio y entornos de enseñanza-aprendizaje, $b$ ) el diseño de programas de estudio glocales, contenidos didácticos, campos de aprendizaje y resultados, y $c$ ) el diseño de ámbitos de enseñanza-aprendizaje glocales con educación en línea, entornos mixtos y formatos colaborativos. Este modelo ha llevado a reflexionar sobre las competencias que es necesario generar en los participantes, las diferencias con los planes y programas tradicionales, y su escala a programas de posgrado (John et al., 2017).

La segunda experiencia corresponde a los Laboratorios del Mundo Real (LMr, Real-World Laboratories) (Wanner et al., 2018; Schäpke et al., 2018), que son uno de los enfoques más recientes en la búsqueda de formatos para conducir iniciativas de inter y transdisciplina, y se caracterizan por: "(i) el enfoque debe ser transdisciplinario y/o participativo, y/o cocreativo; (ii) participar y/o reflexionar a fondo sobre el cambio social; y (iii) reconocer como principio rector al desarrollo sustentable" (Wanner et al., 2018, p. 96). El proyecto EEVA (Wanner et al., 2018) es un LMR que busca fortalecer a los usuarios para liderar la transformación a través de una participación sociopolítica que oriente los procesos sociales en una dirección sostenible (Wanner et al., 2018) y que considere el aprendizaje transformador como una "interacción entre conocimientos, sensibilidades y habilidades" (Schneidewind, 2018, p. 461, en Wanner et al., 2018). Sus programas cuen- tan con participantes de distintos sectores (académico, empresarial, estudiantado, gobierno, sociedad civil), lo cual ha hecho posible incorporar estas experiencias a la educación formal debido a que: a) proporcionan conocimientos teórico-metodológicos al combinar aportes de los diversos participantes; b) fomentan la apreciación de la complejidad del sistema; c) moderan el debate crítico; $d$ ) crean vínculos personales con el contexto y su transformación; e) permiten la autorreflexión, y f) generan un espacio de confianza para el aprendizaje.

Las experiencias descritas se han realizado, en su mayoría, dentro de contextos europeos y urbanos, e incluyen las competencias educativas para la sostenibilidad que proponen Wiek y colaboradores (2011).

En lo que respecta a la Universidad Iberoamericana, en su Plan Estratégico se destacan los retos de los próximos ańos (rumbo al 2030) y se hace mención explícita de la inter y transdisciplina. Su misión consiste en "contribuir al logro de una sociedad más justa, solidaria, libre, incluyente, productiva y pacífica, mediante el poder transformador de la docencia, la investigación, la innovación y la vinculación, en estrecho contacto con la realidad" (Universidad Iberoamericana, 2018, p. 15). La Ibero se destaca por ser un actor social que se compromete a contribuir en la solución de problemas complejos por medio de creatividad e innovación, y conforme a las necesidades sociales en las que se inserta. Por esto, sus funciones de docencia, investigación y vinculación se deben orientar "hacia la atención de los principales problemas de la sociedad desde lo local hasta lo global, con un enfoque inter y transdisciplinario" (Universidad Iberoamericana, 2018, p. 25).

En el año 2018, la Ibero inició el diseño de nuevos tipos de unidades académicas, por lo que comenzó con el Centrus, cuyo objetivo es generar "una verdadera interdisciplinariedad, enfocada en la resolución de problemas concretos desde el diálogo e interacción entre los diversos campos disciplinares y profesionalizantes, e incluso generando un fructífero diálogo de saberes, donde también tuviesen cabida conocimientos ancestrales y tradicionales" (Guevara et al., 2019, p. 61).

92 - La caminos a la inter y transdisciplina de la Universidad Iberoamericana 
Recientemente, la Universidad Iberoamericana lanzó la renovación de sus planes de estudio, denominados Planes Manresa, los cuales fueron desarrollados con base en una visión de interdisciplina, flexibilidad e incidencia social. Igualmente, contemplan asignaturas obligatorias con un pensamiento humanista, en contacto con la realidad, con perspectiva de género y enfocadas hacia la sustentabilidad e interculturalidad (Rendón, 2021). No obstante, queda pendiente evaluar si esta renovación resultó ser un instrumento efectivo para impulsar la inter y transdisciplina en la universidad.

\section{Enfoque y métodos: nuestra aproximación}

La metodología general que empleamos tiene un carácter mixto, lo cual permite el estudio de los fenómenos de interés a partir de instrumentos que buscan a) entender e interpretar dichos fenómenos en términos de los significados que los involucrados les otorgan y b) darles una dimensión cuantitativa a las respuestas de los participantes. Los resultados arrojan dos tipos de datos: los conteos y las frecuencias de las respuestas en las categorías formuladas previamente, y la interpretación de los discursos de las personas entrevistadas que buscan la complementariedad de ambos enfoques (Hernández Sampieri et al., 1991; Sánchez, 2014). Nos aproximamos al tema de la investigación recolectando materiales empíricos sustentados en preguntas y, a partir de éstas, sistematizamos, analizamos y teorizamos los resultados y concluimos (Denzin \& Lincoln, 1994), por lo que predomina el enfoque cualitativo.

Para recabar la información, llevamos a cabo seis entrevistas semiestructuradas que se basaron en una pauta de temas (Harding, 2002). Además, realizamos encuestas a partir de una guía de 11 preguntas más puntuales. Ambos métodos nos permitieron captar datos para estructurar y homogeneizar las preguntas y respuestas (Bernard, 2006; Arce et al., 1998).

Con el objetivo de plasmar la naturaleza colaborativa de la inter y transdisciplina, decidimos que el presente artículo contaría con la participación de los directores de las divisiones de la Ibero, a quienes agradecemos su disposición a ser entrevistados. Cabe mencionar que estas divisiones posibilitan la organización académica de nuestra universidad. Al interior del Organigrama Institucional de la Universidad Iberoamericana Ciudad de México y Tijuana, se encuentra la Vicerrectoría Académica, que vela por la calidad académica de toda la docencia y la investigación, y dentro de esta vicerrectoría se ubican las divisiones que concentran los departamentos, centros e institutos de licenciaturas, carreras técnicas y posgrados que, a su vez, cuentan con sus respectivos coordinadores. De esta estructura organizacional, se eligieron cuatro divisionales, ${ }^{2}$ y al director y coordinadora del Departamento de Reflexión Interdisciplinaria de la Ibero, anteriormente conocido como Área de Reflexión Universitaria (ARU). ${ }^{3}$

Las entrevistas tuvieron una duración promedio de 30 a 60 minutos. Se llevaron a cabo vía Zoom y fueron grabadas y transcritas. El confinamiento ocasionado por la pandemia de COVID-19 ha conllevado a que este tipo de métodos de investigación se desarrolle de manera virtual.

Las entrevistas semiestructuradas se enfocaron en dar conocer la oferta de los programas formativos existentes de vision inter o transdisciplinaria, por medio de las experiencias de los directores de las divisiones de la Ibero. Las entrevistas se dividieron en cinco apartados: el primero sirvió para plasmar una estampa etnográfica ${ }^{4}$ de éstas; el segundo ayudó a conocer la ocupación del participante para situar su vida laboral al interior de la Ibero; en el tercero se indagó acerca de la percepción del participante sobre temas de interdisciplina en la Universidad; el cuarto consistió en averiguar la perspectiva del participante sobre temas de transdisciplina en esta institución, y en el último se reflexionó en torno a las posibles rutas, caminos e ideas del participante para mejorar los programas de inter y transdisciplina en la Universidad, es decir, pensar rumbo al futuro.

Para organizar y sistematizar los datos obtenidos de las entrevistas, usamos la propuesta de codificación de la Teoría Fundamentada (Glaser \& Strauss, 1967), que consiste en la identificación de las características de dichos datos y en la comparación entre 
éstos para encontrar coincidencias y diferencias que permitan analizarlos de manera sistemática.

Una vez que se analizaron estas primeras entrevistas semiestructuradas, levantamos una encuesta de 11 preguntas (seis de opción múltiple, cuatro abiertas y una matriz), ${ }^{5}$ que se envió a los directores y coordinadores de $89^{6}$ programas académicos dentro de la Ibero; 85 de éstos respondieron. Esta encuesta buscó conocer, de primera fuente, las percepciones que se tienen sobre los distintos programas académicos de la Ibero respecto a la disciplina, inter y transdisciplina, para recuperar el panorama actual y complementarlo con el que deseamos construir al interior de la universidad.

En total se recibieron $96^{7}$ respuestas de los académicos que coordinan o participan en la organización de alguno de los siguientes tipos de programas educativos: Técnico Superior Universitario (TSU), licenciatura, especialidad, maestría o doctorado. Cabe mencionar que la Ibero cuenta con 36 programas de licenciatura, 29 de maestría, 11 de doctorado, 6 de especialidad y 7 de Tsu. Las respuestas se vaciaron en una tabla general con 37 variables y 96 observaciones, así como una variable que indica el tipo de programa académico en el que participa la persona encuestada, y otra que señala el área de estudio en el que se agrupó el programa. ${ }^{8}$

Dada la importancia de contar con planes y programas de estudio reconocidos y acreditados por la Secretaría de Educación Pública, se realizó una revisión general de la normatividad aplicable, con la

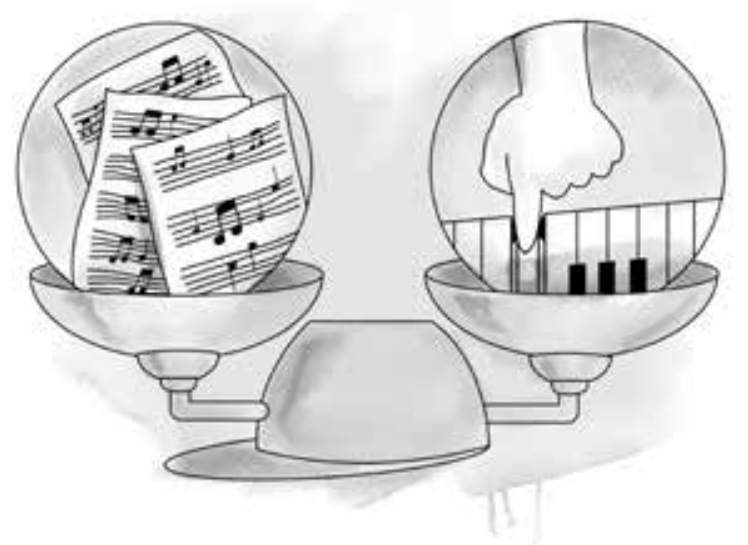

finalidad de comprender en qué términos están referidas la inter y transdisciplina y en qué medida se favorece u obstaculiza su inclusión. ${ }^{9}$

\section{Resultados: nuestros hallazgos a lo largo del camino}

\section{a) Las divisiones frente a las iniciativas de inter y transdisciplina en la Ibero}

A partir de las experiencias relatadas por los directores de las divisiones de la Ibero, presentamos la visión de la oferta de programas educativos que cuentan con un enfoque inter o transdisciplinario y cuyo objetivo es "lograr el funcionamiento idóneo de todos los departamentos académicos [que la conforman] para responder a los objetivos, a la misión y visión de la universidad" (A. Polo, comunicación personal, 10 de diciembre de 2020).

Se han establecido diversas iniciativas para integrar los enfoques inter y transdisciplinarios en la docencia de la universidad, una de las más recientes es el Seminario Permanente de Multi, Inter y Transdisciplina. Se trata de un espacio colaborativo encabezado por un grupo de académicas, académicos y personal directivo de la Ibero y que, en sus últimas fases, permitió "la creación de seis grupos que avanzaron de forma independiente hacia sus objetivos"; ${ }^{10}$ este espacio se ha enfocado en el diseño de programas académicos articulados en torno a problemáticas actuales. Una de las actividades ejecutadas en este seminario, en Otoño de 2019, fue realizar un taller sobre inter y transdisciplina que contó con dos participantes internacionales: Ulli Vilsmaier, de la Universidad Leuphana de Lüneburg, Alemania, y Dina Fam, de la Universidad Tecnológica de Sydney, lo que generó encuentros de diálogo y reflexión teóricoconceptuales sobre los esquemas de docencia universitaria que favorecen la inter y transdisciplina. Sin embargo, está pendiente que este seminario se adapte al contexto de la Ibero.

La vicerrectoría ha impulsado el interés por diseñar nuevos planes de estudio que, desde su inicio, se conciban como inter y transdisciplinarios, que respondan a las necesidades actuales y se instauren dentro de los tres ejes temáticos de interculturalidad,

94 - La caminos a la inter y transdisciplina de la Universidad Iberoamericana Celia Arteaga Conde, José Alberto Lara Pulido, José Alberto Gallardo Cruz, Carmina Isabel Jiménez Quiroga, Adriana Carolina Flores Díaz, Raúl Gutiérrez Patiño, Juan Manuel Núñez. DIDAC 78 (2021): 88-107 
asuntos de género y sustentabilidad. En este ámbito también se encuentran las cátedras universitarias interdisciplinarias que son iniciativas de investigación en donde participa el profesorado de diferentes departamentos y cuyo objetivo es estudiar un problema complejo común y que suelen incluir, en general, la participación de estudiantes de diversas áreas.

Por su parte, el Departamento de Reflexión Interdisciplinar (creado en 2020), antes Programa de Reflexión Universitaria (ARU), constituye otra iniciativa para pensar en torno a estos enfoques, ya que provee un espacio docente para el encuentro del estudiantado de diversas carreras; en éste se reflexiona acerca de problemáticas sociales actuales.

Respecto a la interdisciplina, todas las carreras de los planes vigentes (Planes suj) cuentan con materias departamentales, tanto obligatorias como optativas, en las que participan diferentes licenciaturas que nutren los departamentos académicos para que los alumnos cuenten con "una visión integral [...] que les [brinde] las competencias necesarias para ver el fenómeno de otra manera" (A. Polo, comunicación personal, diciembre de 2020). También están las materias de reflexión universitaria, donde el estudiantado de diversas licenciaturas analiza un mismo problema. Las materias ARU, por tanto, tienen como objetivo "la formación integral en todos los planes de estudios de licenciatura" (Universidad Iberoamericana, 2020, p. 11). Además, están las materias de servicio social, que buscan que el alumnado se aproxime al campo con una visión interdisciplinaria. El Departamento de Reflexión Interdisciplinaria cuenta con el Seminario Thizy por el Bien Común, donde se plantea la posibilidad de reflexionar y de hacer investigaciones en torno al bien común: ${ }^{11}$ "Procuramos estar más atentos en función de las necesidades, proyectos, alternativas, posibilidades que podemos tener con académicos, académicas, alumnos, alumnas, no solamente al interior de la Ibero, sino a un nivel nacional o fuera del país" (J. F. de la Fuente, comunicación personal, febrero de 2021).

$\mathrm{Al}$ interior de la División de Ciencia, Arte y Tecnología, se han llevado a cabo algunas actividades puntuales. El Departamento de Ingeniería Elec- trónica trabajó de la mano con el de Historia (que pertenece a la División de Humanidades y Comunicación) en la digitalización del trabajo de Edmundo O’ Gorman (siglo XviI novohispano). En conjunto, estos departamentos desarrollaron un disco interactivo. Por su parte, en la licenciatura en Ingeniería de Alimentos se puede advertir la perspectiva interdisciplinaria en la investigación de las ciencias básicas y aplicadas para la solución de problemas. En este sentido, la fusión de Ingeniería en Tecnologías de Cómputo y Telecomunicaciones es un intento por hacer interdisciplina. En esta división se identifican proyectos transdisciplinarios, por ejemplo: $I$ ) el Departamento de Arquitectura se comunica con diversas áreas al interior y fuera de la universidad para resolver proyectos como la construcción de carreteras, calles o azoteas verdes, en los cuales "tienen que entender la energía, los huertos urbanos, la ciencia energética y las ciudades inteligentes" (J. Morfín, comunicación personal, diciembre de 2020); II) en el de Diseño, el uso de tecnología es fundamental; la licenciatura en Ingeniería Mecatrónica estudia la convergencia de Ingeniería Mecánica, Sistemas Mecánicos, Electrónicos y de Programación. El Centro Transdisciplinar Universitario para la Sustentabilidad (Centrus) se diseñó "para trabajar un tema en particular desde diferentes disciplinas, en este caso es el de la sustentabilidad" (J. Morfín, comunicación personal, diciembre de 2020). Dentro de este centro se instaura la licenciatura en Sustentabilidad Ambiental (Lisa), que agrupa a docentes de múltiples disciplinas como Antropología, Biología, Ciencias Políticas, Derecho, Economía, Ingeniería, entre otras.

En lo que respecta a la División de Humanidades y Comunicación, ésta cuenta con varios proyectos dentro de sus departamentos. En Historia, se digitalizaron archivos municipales y parroquiales y se capacitó al personal para promover procesos de la memoria histórica; Letras, por su parte, tiene proyectos de alfabetización; en Arte hay proyectos de acceso universal a la cultura, por ejemplo, se han transportado piezas de diversos museos a zonas marginales y penitenciarías; Ciencias Religiosas posee centros de acción parroquial con seminarios y cen- 
tros de acción social eclesiásticos. Acerca de todos estos proyectos "se cierra el ciclo; traemos conocimiento que se refleja en investigaciones que sirven eventualmente para consolidar carreras de investigación y, por tanto, se reflejan en libros, en papers, en comunicaciones" (J. Cuesta, comunicación personal, diciembre de 2020).

La División de Investigación y Posgrado, por su parte, favorece que los proyectos de investigación sean transdisciplinarios y que incluyan a diferentes departamentos. Destaca el proyecto de milpas educativas en donde "ya no es solamente venir a la escuela separada de la comunidad y separada de la vida y de la vivienda y del hogar" (M. Silva, comunicación personal, enero de 2021). Asimismo, existe un proyecto de interculturalidad con comunidades indígenas para generar conocimiento que ayude a transformar sus condiciones de vida.

Por otro lado, en la División de Estudios Sociales se encuentra la maestría en Migración, que procura vincularse con otras redes como las universidades jesuitas y con otros espacios que abordan desde distintos frentes el fenómeno migratorio, lo que resulta un campo claramente interdisciplinar, ya que busca que su núcleo docente sea interdisciplinar. Cuenta con tres ejes: una base teórico-metodológica donde se diseñan y evalúan políticas públicas, programas y proyectos de intervención; uno de conocimiento especializado para abordar la problemática migratoria, y finalmente, el de investigación para que los estudiantes desarrollen un proyecto de investigación que atienda "problemáticas derivadas de la gestión migratoria y, a que [...] realicen estudios de campo" (Maestría en Estudios sobre Migración). Alma Polo plantea que los programas interdisciplinarios, como esta maestría, abordan los problemas

desde diferentes lentes y eso te abre más puertas para poder hacer intervención social, [pues ésa] es la idea de tener posicionamiento y presencia en políticas públicas a partir de la visión de diferentes disciplinas para ayudar a los gobiernos a dar soluciones. (A. Polo, comunicación personal, diciembre de 2020).
Otro ejemplo es el Doctorado de Estudios Críticos de Género (DECG), pues en él converge profesorado de distintas disciplinas como Arte, Ciencias Sociales y Políticas, Ciencias Religiosas, Comunicación, Derecho, Desarrollo Humano, Economía, Estudios Internacionales, Filosofía, Literatura, Historia, Psicología, entre otras, para analizar fenómenos relacionados con el género. Este doctorado incluye un semestre de investigación en incidencia social, lo cual representa para el alumnado una guía en la elaboración de "propuestas de investigación e incidencia social [para] plantear problemáticas de género actuales desde un punto de vista crítico" (DECG, 2017).

En lo concerniente a las acciones transdisciplinarias mencionadas por los participantes, al interior de la universidad destacan las prácticas profesionales cuyo objetivo es aplicar los conocimientos adquiridos dentro de las aulas, ya que su modelo es el "aprendizaje y servicio: lo que tú aprendes, lo puedes contribuir a la sociedad" (A. Polo, comunicación personal, diciembre de 2020). Estas prácticas enriquecen la formación integral del estudiantado al complementar los aprendizajes académicos aplicándolos al contexto actual. La variedad académica que se ofrece desde educación continua es otro ejemplo de iniciativa transdisciplinar.

Dentro de los espacios universitarios que emprenden acciones encaminadas a la inter y transdisciplina, los participantes identificaron a los comités de Ecología, Derechos Humanos y Género. ${ }^{12}$ Éstos son percibidos como trabajos transdisciplinares puesto que constituyen alternativas de "estudio y reflexión en torno a temas fundamentales e interdisciplinarias o transversales" (J. F. de la Fuente, comunicación personal, febrero de 2021).

Adicionalmente, los integrantes del Senado Universitario se han acercado a los departamentos para difundir su labor y tener una conversación sobre las preferencias apostólicas de la Compañía de Jesús. Según uno de sus miembros, José Luis Gutiérrez Brézmes, la visión del senado es transdisciplinar, ya que es "un ente orgánico con muchas partes que interactúan entre sî" (comunicación personal, abril

96 - La caminos a la inter y transdisciplina de la Universidad Iberoamericana Celia Arteaga Conde, José Alberto Lara Pulido, José Alberto Gallardo Cruz, Carmina Isabel Jiménez Quiroga, Adriana Carolina Flores Díaz, Raúl Gutiérrez Patiño, Juan Manuel Núñez. DIDAC 78 (2021): 88-107 
de 2021) donde la Universidad intenta abarcar el universo del conocimiento. Se debe tener la suficiente amplitud de visión y criterio para acudir a personas que piensen diferente $y$, construir, por medio del diálogo y la voluntad comunes, saberes que reflejen el quehacer transdisciplinario.

Finalmente, los entrevistados coincidieron en que tanto el Centrus como la Lisa y el DECG son los programas pioneros, es decir, los referentes de la inter y transdisciplina en la universidad.

\section{b) Los enfoques inter y transdisciplinar dentro de los programas de la Ibero}

En la primera pregunta se pedía a la persona encuestada que indicara qué tanto el nombre del programa que coordina refiere un tema disciplinar. Al respecto, $40 \%$ respondió que mucho, otro $40 \%$ que poco y $20 \%$ que nada $(n=96)$. Cuando revisamos las respuestas por orientación disciplinar, notamos que las ciencias sociales tienden a concebir el nombre de sus programas como poco disciplinares ( $48 \%$ eligieron "poco" y $13 \%$, "nada"), al igual que las ingenierías (41\% optaron por "poco"). En contraste, el grupo de estudios empresariales los considera muy disciplinares (53\% seleccionaron la categoría "mucho"). Estos datos sugieren que son los programas enfocados en el estudio de la empresa los que más aluden a ésta como un campo acotado de estudio, en cambio, los nombres de programas de ciencias sociales y de ingenierías evocan en los participantes un espectro más amplio del conocimiento. En este sentido, conforme a la visión de los encuestados, los nombres de los programas sugerirían a los estudiantes que desean abordar temas que trascienden las barreras de una disciplina inclinarse por la oferta educativa de las ciencias sociales o las ingenierías.

A nivel del grado académico, para los doctorados y las licenciaturas las respuestas indican que los nombres de los programas hacen "mucha" referencia a un tema disciplinar ( $54 \%$ y $46 \%$, respectivamente); las especialidades, por su parte, mantienen una distribución equitativa entre "mucho" y "poco" (44\% y $33 \%$, respectivamente); las maestrías son las que muestran mayor concentración en la respuesta "poco"
(54\%). En las Tsu, la distribución es del 33\% para cada respuesta. La mayoría de los académicos $(60 \%)$, que no son coordinadores, opina que el nombre del programa en el que participa refiere en gran medida a un tema disciplinar. Lo anterior arroja conclusiones encontradas. Los nombres de los doctorados, programas con el mayor nivel de especialización, transmiten un alto grado de disciplinariedad, mientras que los de las maestrías (nivel de especialización anterior al doctorado) se conciben como programas que aluden muy poco a una disciplina específica, al igual que las licenciaturas, que son programas de profesionalización mas no de especialización. Así pues, no es sino hasta el nivel de doctorado que los nombres de los programas refieren temas disciplinares, quizá porque en éste se abordan contenidos muy específicos dentro de una disciplina o ciencia.

En las preguntas siguientes se pidió a los encuestados indicar el enfoque del programa respecto a las categorías: disciplinar, multidisciplinar, interdisciplinar y transdisciplinar, donde las opciones de respuesta eran "mucho", "poco" y "nada". La Gráfica 1 muestra la frecuencia de cada respuesta respecto a cada atributo. La menor cantidad de respuestas corresponde a programas con un enfoque transdisciplinar. En contraste, la mayoría percibe en los programas de la Ibero un enfoque disciplinar, pues 62 de los 96 encuestados consideran que su programa mantiene dicha perspectiva. A esta perspectiva le sigue la interdisciplinar con 52 respuesta para "mucho".

Cabe mencionar que $62 \%$ de los participantes considera que el enfoque de su programa es disciplinar, comparado con $40 \%$ que percibe que el nombre de su programa alude a un tema disciplinar; por tanto, más del $20 \%$ de los encuestados estima que el nombre de su programa no transmite el tipo de enfoque que en verdad posee.

En cuanto al cruce de las respuestas, de 29 participantes que respondieron "mucho" al atributo transdisciplinar, 15 también consideraron que su programa es muy disciplinar, 18 que es muy multidisciplinar, y 25, muy interdisciplinar. Por lo anterior, se infiere que, en general, los encuestados tienden a percibir que sus programas son muy transdisciplinares (29) e inter- 
disciplinares, y, en menor medida, los conciben como multidisciplinares; aunque un $50 \%$ considera que también son disciplinares. En suma, se percibe que la transdisciplina coexiste mejor con la interdisciplina.

Gráfica 1

Frecuencia de aparición por respuesta (mucho, poco, nada) para describir el enfoque del programa ( $n=96)$

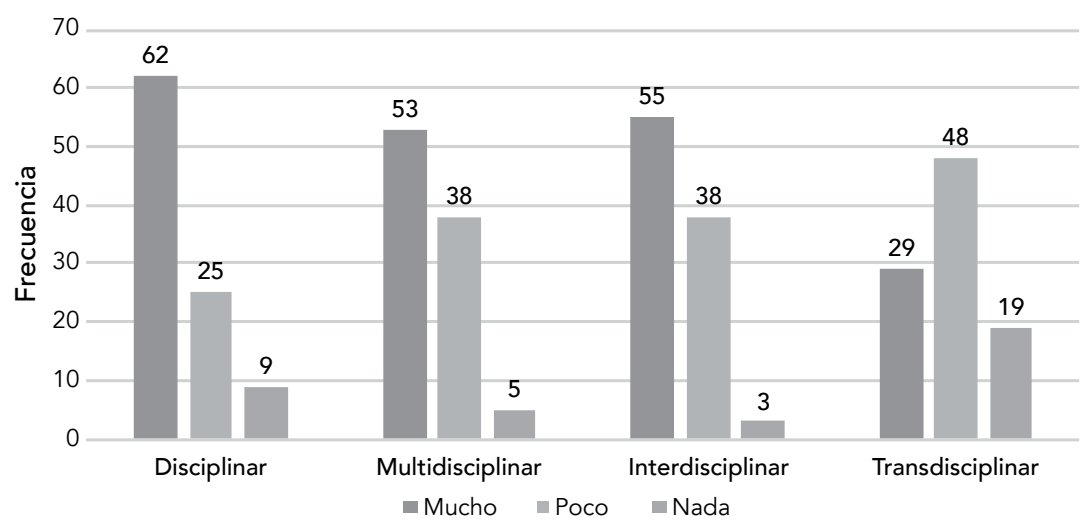

Fuente: Elaboración propia

Respecto al rubro transdisciplinar, 30\% de los encuestados respondió "mucho" y sólo $20 \%$ "nada”. De las licenciaturas, $28 \%$ respondió "mucho", al igual que el $45 \%$ de maestría, el $18 \%$ de doctorado, el $33 \%$ de especialidades y $17 \%$ de Tsu. Por área de estudio, las que alcanzan un mayor porcentaje de respuestas "mucho" son las empresariales, religiosas, ambientales y de la salud, con aproximadamente
$50 \%$. En el área de estudios religiosos y ciencias ambientales, sólo hubo dos personas encuestadas, y en el área de ciencias de la salud hay 4, por lo que estas respuestas podrían estar sobrerrepresentadas. En el área de las artes, nadie respondió "mucho". En las categorías de diseño, sociales e ingenierías, menos del $35 \%$ de los encuestados contestó "mucho" (Véase Gráfica 2).

Gráfica 2

Porcentaje de personas encuestadas por área de estudio que consideran que el enfoque de su programa es transdisciplinar dentro de la categorí "mucho" $(n=96)$

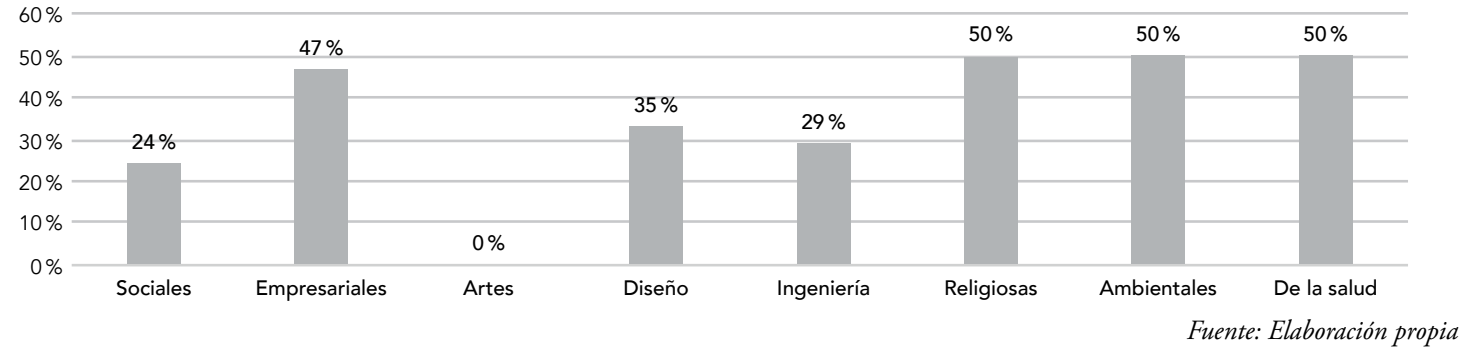

Así pues, existe congruencia en los programas de maestría, ya que los encuestados consideran que el nombre no evoca un tema disciplinar mientras que el enfoque es calificado como muy transdisciplinar. Lo mismo sucede con los doctorados, pero en sentido opuesto, es decir, se perciben como programas disciplinares. Las licenciaturas, igualmente, parecen concebirse como programas más disciplinares que transdisciplinares, sin embargo, la concentración de las respuestas resulta menos evidente. Respecto a las áreas del conocimiento, en estudios empresariales se presenta una contradicción, puesto que se considera, en su mayoría, que el nombre de su programa alude a un tema disciplinar, pese a que el enfoque de sus programas es transdisciplinar. Lo mismo sucede con los programas de ciencias sociales, aunque en sentido contrario, pues el nombre de los programas refiere poco a temas disciplinares, no obstante, el enfoque es

98 - La caminos a la inter y transdisciplina de la Universidad Iberoamericana 
disciplinar. En este sentido, existe una discrepancia entre cómo se perciben los nombres de los programas y sus contenidos.

También se cuestionó de qué manera el programa académico presenta un enfoque inter o transdisciplinar. La Gráfica 3 muestra el número de veces que se eligió cada una de las opciones ( $\mathrm{n}=96$, se podía optar por más de una opción), dentro de las categorías de respuestas preestablecidas. La mayoría considera que su programa integra este enfoque a través de sus planes de estudio, ya sea con algunos créditos o en la totalidad del plan. Las prácticas profesionales y el servicio social le siguen en importancia. Sólo en 30 respuestas se muestra que en su programa se llevan a cabo intercambios de profesores con otros departamentos. Por otro lado, dos consideran que su programa no integra este enfoque en ninguna forma; y la mayoría (72\%) seleccionó más de una de las opciones.

Gráfica 3

Formas en las que el programa implementa un enfoque transdisciplinar (frecuencia) $(n=96)$

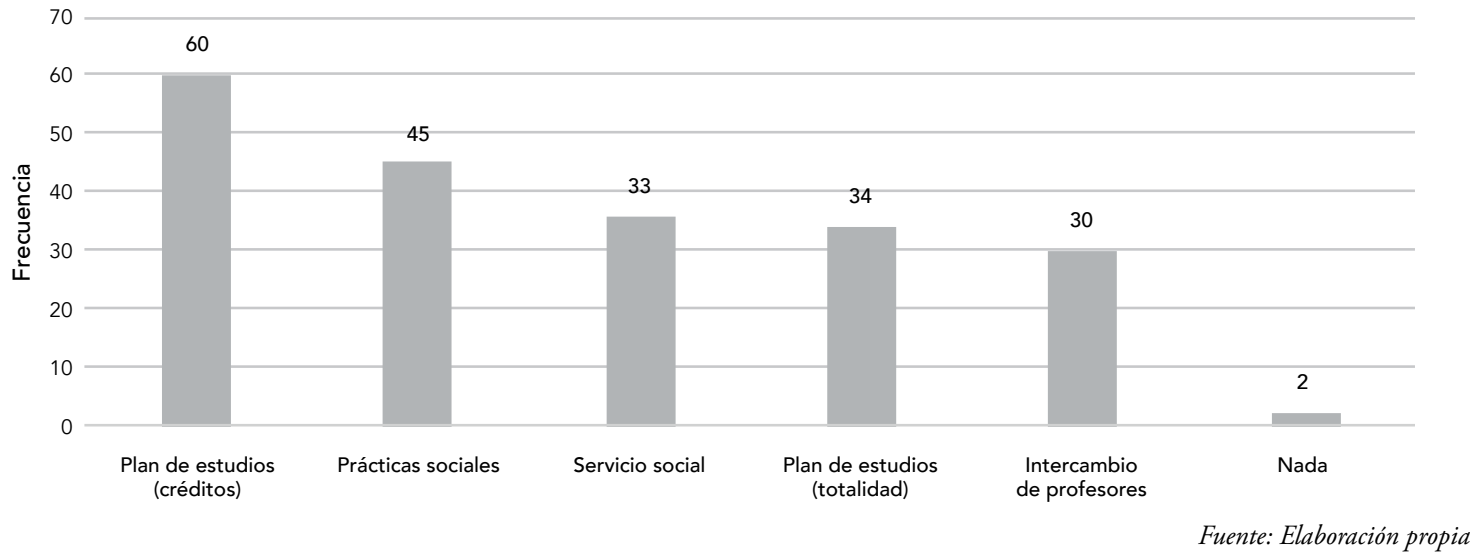

El 69\% de los programas participantes comparten materias con otros. Este porcentaje sube a $76 \%$ en licenciatura, a $78 \%$ en los de doctorado y a $83 \%$ en los de TSU. Los programas de maestría, por su parte, presentan el porcentaje más bajo, con un $50 \%$. Respecto al área de estudio, los religiosos son los que más comporten materias con otros programas ( $100 \%$, aunque hay que recordar que son sólo dos observaciones), seguidos por los empresariales y diseño ( $77 \%$ cada uno), artes y ciencias de la salud ( $75 \%$ cada uno); después se encuentran los de ciencias sociales $(67 \%)$ e ingeniería (58\%).

A partir de los datos de estas preguntas, la percepción es que la transdisciplina se incluye en la docencia de la Ibero a través de materias compartidas con otros programas (que forman parte de los planes de estudio), y que son recurrentes en la mayoría de éstos, sin importar el grado académico ni el área del conocimiento.

El $93 \%$ opina que la interdisciplina enriquecería mucho su programa, debido a: a) el abordaje de problemáticas o fenómenos complejos, $b$ ) que los límites disciplinares son insuficientes, $c$ ) la naturaleza interdisciplinar del campo de estudio, $d$ ) la importancia de incluir otros enfoques o puntos de vista que enriquecen el aprendizaje y la práctica, e) que genera un conocimiento integral, $f$ ) la necesidad de ampliar la visión, $g$ ) que el diálogo entre saberes construye y enriquece, y h) que la vida laboral es interdisciplinar. Otras respuestas refirieron la idea de una mirada crítica, el intercambio de experiencias, la importancia de estudiar algo desde fuera y a partir de la diversidad.

La interdisciplina es referida por los participantes como una buena propuesta para generar e intercambiar conocimientos de manera colaborativa que, además, podría traer múltiples ventajas al interior de los programas formativos con los que cuenta la Ibero. En suma, permitiría cruzar los límites disciplinarios del conocimiento con base en un diálogo horizontal.

El $79 \%$ de los encuestados considera que la transdisciplina enriquecería su programa, 19\% que 
lo enriquecería poco y $2 \%$, nada. Los que respondieron "mucho" hablaron sobre la importancia de conocer a los beneficiarios de las soluciones que ofrece cada disciplina y de integrar visiones no académicas en la generación de conocimiento compartido, así como del papel fundamental de las visiones de quienes viven una realidad específica en el abordaje de cualquier problemática. Se hizo énfasis en la palabra "realidad" y en la idea de que el conocimiento académico debe aterrizarse a contextos específicos. En general, se percibe a la transdisciplina como una vía para generar impacto e incidencia. Cabe señalar que algunas respuestas refieren cuestiones interdisciplinares más que a transdisciplinares, de acuerdo con la definición expuesta al inicio de este texto.

Dentro de la visión de los participantes, la transdisciplina permite la construcción e intercambio de experiencias diversas con actores ajenos a la academia, lo que favorece las oportunidades del alumnado para atender problemáticas y necesidades actuales. Éste y otros conceptos relativos a la transdisciplina necesitan ser revisados con una base conceptual sólida, con la finalidad de contar con una demarcación clara de cada enfoque.

Finalmente, se preguntó de qué manera la inter y transdisciplina podría funcionar mejor en la Ibero. Así, 23 participantes mencionaron la necesidad de crear espacios de diálogo e intercambio entre diferentes departamentos y áreas de la universidad, para que exista un mayor grado de articulación y cooperación dentro de la labor educativa. Del total de encuestados, 11 señalaron que una buena forma de integrar estos conceptos es generar proyectos que pongan en contacto al alumnado con la realidad, la industria, las empresas, el sector público, entre otros, así como que se construyan conjuntamente entre departamentos. De igual manera, fue recurrente (alrededor de ocho respuestas) la idea de crear materias que pertenezcan a más de un departamento o que sean diseñadas por personal académico de diferentes departamentos y en las cuales se aborden problemas complejos de carácter inter y transdisciplinar. Aproximadamente ocho personas consideran que las trabas burocráticas y administrativas son un reto importante para la transdis- ciplina en la Ibero, por lo que es necesario flexibilizar procesos. Por último se indica, en al menos ocho ocasiones, la relevancia de introducir actores no académicos como parte de la cátedra de la universidad para establecer espacios de diálogo con otros actores.

Es importante continuar con la reflexión en torno a las definiciones conceptuales de la inter y la transdisciplina, ya que para los participantes la frontera entre ambas no resulta muy clara.

\section{c) Consideraciones de la normatividad nacional para la formación integral a través de la inter y transdisciplina}

La búsqueda de la formación profesional del alumnado nos lleva a reflexionar sobre los alcances de los planes de estudio en favor de su desarrollo como seres humanos y en su consolidación como agentes de cambio, lo cual representa una misión de las universidades según las disposiciones de la fracción VII, artículo $3 .^{\circ}$, de la Constitución Política de los Estados Unidos Mexicanos. Asimismo, el artículo 7. ${ }^{\circ}$ de la Ley General de la Educación Superior, publicada recientemente, establece que la educación superior "fomentará el desarrollo humano integral del estudiante en la construcción de saberes".

La formación integral constituye el desarrollo armónico de todas las dimensiones de la persona: psicoafectiva, sociocultural, intelectual, artística, entre otras. En ese sentido, resulta necesario que los programas de estudio cuenten con enfoques pedagógicos que la promuevan. Si bien la legislación no apunta específicamente hacia la inter o transdisciplina como aspectos normativos para lograr tal efecto, esto se advierte con base en su interpretación, la educación ha de ser más efectiva, es decir, verdaderamente integral, por tanto, debe favorecerse que el estudiantado sea capaz, por medio de un acompañamiento del profesorado, de captar y de apropiarse de la relación entre las diferentes disciplinas para notar cómo se influyen y enriquecen mutuamente, además de considerar los contenidos, valores y actitudes para interpretar la realidad personal y social desde una perspectiva más compleja. De ahí que sea preciso concebir la formación integral como inter y transdis-

100 - La caminos a la inter y transdisciplina de la Universidad Iberoamericana Celia Arteaga Conde, José Alberto Lara Pulido, José Alberto Gallardo Cruz, Carmina Isabel Jiménez Quiroga, Adriana Carolina Flores Díaz, Raúl Gutiérrez Patiño, Juan Manuel Núñez. DIDAC 78 (2021): 88-107 
ciplinar, para consolidarla al interior de los programas de estudio y en el proceso de acreditación.

$\mathrm{Al}$ respecto, debe tomarse en cuenta la importancia que representa la acreditación de los programas de estudio de educación superior avalados por instituciones externas como el Consejo para la Acreditación de la Educación Superior, A. C. (COPAEs), lo cual se fortalece debido a los compromisos internacionales del Estado mexicano plasmados ante la Conferencia Regional de la Educación Superior en América Latina, en donde se recomendó a las áreas correspondientes de los gobiernos consolidar los sistemas nacionales de acreditación y evaluación y apoyar la construcción e implementación de sistemas de autoevaluación en las Instituciones de Educación Superior (IES). Asimismo, se les ha recomendado trabajar en la búsqueda de un continuo mejoramiento en el cumplimiento de sus misiones institucionales y fomentar la cultura de la evaluación y el compromiso con los sistemas de evaluación y acreditación (Castillo et al., 2014, en Rueda, 2009).

\section{Discusión: nuestros puntos de anclaje}

Proponemos una hoja de ruta para determinar hacia dónde se debe continuar, así como los retos y las propuestas que buscan encaminar las distintas iniciativas de la vida académica de la Ibero rumbo a la construcción de comunidades de aprendizaje bajo los enfoques inter y transdisciplinarios.

En principio, es importante construir un lenguaje común que, por medio de la apertura y flexibilidad, abra el diálogo conjunto para que no se pierda el sentido y uso de la inter y la transdisciplina:

no podemos permitir que conceptos y principios fundamentales para la visión de la universidad empiecen a perder el sentido o a desgastarse. Es sustancial convocar al diálogo, comunicar información precisa para saber de qué estamos hablando y que no se vacíe de contenido. (M. Silva, comunicación personal, enero de 2021).

Para generar este diálogo, deben existir condiciones institucionales que fomenten el trabajo colabora- tivo. Una de éstas es contar con una clara asignación de tiempo para esta colaboración, de forma que los investigadores tengan modo de balancear sus actividades de investigación, docencia, divulgación, difusión y gestión. Dicha reflexión se orienta en contar con respaldo y reconocimiento institucional para realizar estas tareas; por ejemplo, que el formulario cuente con un apartado de las funciones anuales que cada académico ejerce. Asimismo, puede pensarse en un sistema de incentivos para el trabajo colaborativo, como la descarga de horas de otras actividades, reconocimientos, acceso a fondos $\mathrm{u}$ otros mecanismos que generen un interés para llevar a cabo este trabajo.

Otras acciones que podrían favorecer las condiciones para el trabajo colaborativo serían crear espacios específicos para la reflexión, es decir, estructuras internas que se encarguen de ello. Javier Cuesta destaca que la Universidad Nacional Autónoma de México (UNAM) cuenta con el Instituto de Investigaciones sobre la Universidad y la Educación (IISUE) que genera y difunde conocimientos sobre la UNAM, con lo que se constituye un espacio para reflexionar sobre el propio actuar y para señalar puntos de contacto entre diversos departamentos, lo que contribuye a potenciar el trabajo colaborativo. Además, es necesario recuperar espacios atractivos y accesibles que fomenten la comunicación (huerto urbano, cafeterías y otros puntos de encuentro), detonen el diálogo y engendren múltiples intercambios que al mismo tiempo ayuden a la integración universitaria.

Luego de tener claro lo que se pretende lograr, se debe demostrar a más personas de que estas formas de construir conocimiento dan buenos resultados, pues "lo importante es ir convirtiendo gente y para eso necesitamos evangelizar, para eso necesitamos ir predicando" (J. Cuesta, comunicación personal, diciembre de 2020), es decir, superar las resistencias a trabajar bajo los enfoques inter y transdisciplinarios.

Lo siguiente es fomentar actividades concretas como talleres, cursos y seminarios intensivos, donde colaboren personas de distintas disciplinas y de espacios más allá del académico para generar nuevos conocimientos. Para esto, se deben introducir in- 
centivos como descarga de horas, reconocimientos o fondos.

A la par, se debe fortalecer la difusión de los logros y de los programas inter y transdisciplinarios a toda la comunidad universitaria. Por ejemplo, Alma Polo propone que sea por medio de boletines y logros concretos, además de publicar dichos resultados en revistas, particularmente en "revistas especializadas de alto prestigio", como mencionó Marisol Silva. Por otro lado, debe haber divulgación a nivel interno y externo, por lo cual deben usarse los medios con los que cuenta la Ibero, como "radio Ibero, un área de televisión, redes, yo creo que sí tenemos que tomarnos muy en serio el incidir en la opinión pública" (M. Silva, comunicación personal, enero de 2021).

Por último, José Morfín propone la toma de indicadores y medidas, ya que "si no medimos no podemos saber lo que está pasando" (Comunicación personal, diciembre de 2020). Por tanto, se debe contar con órganos efectivos de regulación, cumplimiento y un acompañamiento puntual, así como implementar métricas, indicadores y evaluaciones, además de rendir cuentas de manera transparente y con resultados concretos. Siguiendo lo reportado por otras experiencias, en el camino de la inter y transdisciplina son importantes tanto la generación de conocimiento como aquellos resultados referidos a la construcción de la propia colaboración, de modo que los indicadores deben atender ambos aspectos (Merçon et al., 2018).

\section{Nuestras propuestas}

En este apartado sintetizamos cuatro propuestas concretas para continuar impulsando los caminos hacia la inter y transdisciplina en la Universidad Iberoamericana.

La primera propuesta se encuentra en un nivel de participación y acción, por lo que es deseable el involucramiento de todas las áreas que conforman la universidad. Para tomar el rumbo de la inter y la transdisciplina, se debe favorecer la convivencia dentro de la pluralidad de enfoques, así como mantener los espacios, los proyectos, las materias y los ejercicios meramente disciplinarios junto a los inter $y$ transdisciplinarios. Estos mecanismos de soporte pueden tomar diversas formas, como incentivos al trabajo colaborativo (descarga de horas de otras actividades, reconocimientos y acceso a fondos de investigación o bonos, etcétera).

La segunda se relaciona con un diálogo a nivel conceptual acerca de lo que entendemos por inter y transdisciplina, pues de las entrevistas y encuestas realizadas surgen diversas formas para entender y demarcar estos enfoques. Estos procesos pueden estar conducidos desde diversas instancias, como se ha hecho desde la Vicerrectoría Académica o de la División de Humanidades y Comunicación. La apertura a espacios de conversación y colaboración, creados para nutrir la inter y transdisciplina, pueden ser tan diversos como los intereses, las necesidades y los vínculos de sus participantes. La apertura a la participación de distintas personas y la convergencia de diversos saberes pueden funcionar como semilleros de actividades y programas innovadores que sirvan para fortalecer los vínculos externos e instaurar a la Ibero como un espacio socialmente abierto. En este tenor, resulta crucial el fortalecimiento de las competencias requeridas tanto en los docentes como en el alumnado, para atender los complejos problemas sociales, por ejemplo, los relacionados con la sustentabilidad (Wiek et al., 2011). Para esto, deben retomarse las experiencias de docencia transdisciplinar y el trabajo del Programa Universitario hacia la Sustentabilidad. ${ }^{13}$ La riqueza de este tipo de iniciativas radica en pensar en la docencia fuera de las aulas, es decir, como espacios transversales conducidos de forma flexible en los que se recuperan tanto los aprendizajes del ámbito de estudio como aquellos referidos a la colaboración.

La tercera apela a la necesidad de contar con mayor flexibilidad en los procesos administrativos al interior de la Ibero y a realizar las adecuaciones necesarias en la asignación de funciones del personal académico para que exista una colaboración en proyectos inter y transdisciplinarios $y$, por consiguiente, se instaure como una labor institucionalizada. Lo anterior requiere el involucramiento de las áreas administrativas. Asimismo, este esfuerzo, seguramente precisa-

102 - La caminos a la inter y transdisciplina de la Universidad Iberoamericana Celia Arteaga Conde, José Alberto Lara Pulido, José Alberto Gallardo Cruz, Carmina Isabel Jiménez Quiroga, Adriana Carolina Flores Díaz, Raúl Gutiérrez Patiño, Juan Manuel Núñez. DIDAC 78 (2021): 88-107 
rá recursos humanos y financieros orientados hacia los nuevos enfoques, por lo que es importante que estas áreas comprendan la importancia de esta iniciativa y participen activamente en la formulación de mecanismos que conduzcan a la transformación de la universidad.

En el área de Servicios Escolares, por ejemplo, deben flexibilizarse los procesos administrativos para facilitar la inscripción del alumnado en materias compartidas por distintos programas académicos, con el fin de lograr una mayor articulación y cooperación en la labor educativa. Cabe señalar que esto es parte de los nuevos programas de estudio denominados Manresa, por tanto, el reto será la implementación y logro de este objetivo. Estos planes también tienen el propósito de incidir en la formación social del alumnado por medio de un taller de integración universitaria, una asignatura de inmersión y cuatro asignaturas obligatorias adicionales, además de la participación, al concluir su carrera, en un proyecto dentro del servicio social, realizando una reflexión de cierre crítica.

Por otro lado, se debe puntualizar que hay programas y proyectos que atienden a un mercado y a las necesidades existentes, por ejemplo, la licenciatura en Sustentabilidad Ambiental, el doctorado en Estudios Críticos de Género y la especialidad en Migración. No obstante, existen otras iniciativas que requieren más apoyo, lo cual se traduce en brindar facilidades al cuerpo académico y que esto se les reconozca como parte de sus funciones, es decir, dosificar su carga de trabajo para que haya espacios y tiempo para su colaboración. Es importante mantener los proyectos y programas disciplinares, así como reconocer su relevancia en un marco más amplio que trasciende sus fronteras disciplinares.

La cuarta propuesta consiste en acreditar y certificar nuevos programas inter o transdisciplinarios, lo cual exige que se cuente con estructuras curriculares flexibles. La Secretaría de Educación Pública (SEP), encargada de otorgar validez oficial a los estudios, se basa en la Clasificación mexicana de planes de estudio por campos de formación académica del INEGI, donde, además de los campos disciplinarios de Edu- cación, Artes y Humanidades, Ciencias Sociales, Administración y Derecho, Ciencias Naturales, Exactas y de la Computación, Ingeniería, Manufactura y Construcción, Agronomía y Veterinaria, Salud y Servicios, se plantean programas multidisciplinarios o generales que incluyen los que se conforman por dos o más campos de estudio, motivo por el cual no pueden clasificarse por el criterio dominante.

\section{Gráfica 4 \\ Propuestas para impulsar la inter y transdisciplina}

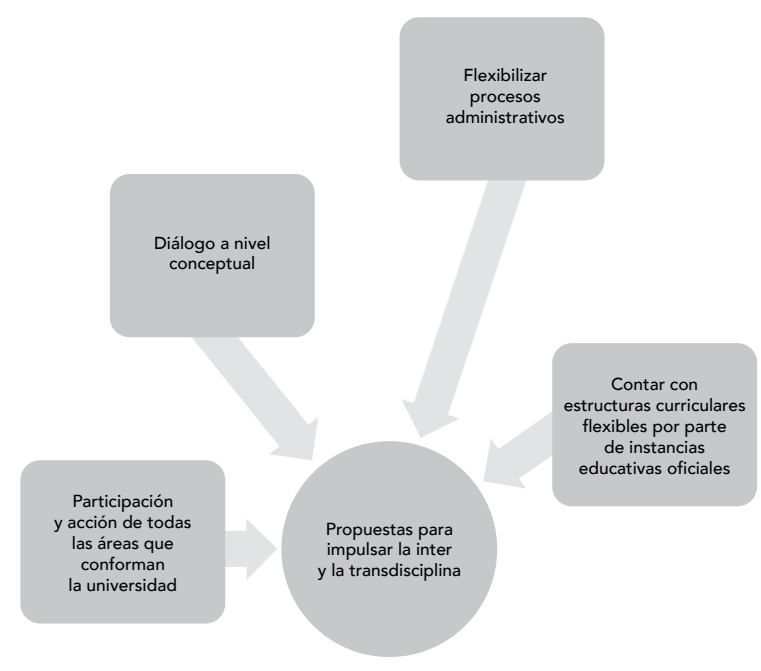

Fuente: elaboración propia

\section{Conclusiones: nuestra reflexión}

Una vez esbozado el diagnóstico sobre la oferta de los programas educativos que tienen una visión inter y transdisciplinaria en la universidad, así como una hoja de ruta, planteamos una serie de reflexiones finales encaminadas a la construcción de una comunidad de aprendizaje.

Los lineamientos institucionales deben sentar la guía de construcción de los enfoques inter y transdisciplinarios, para que abran canales de comunicación y diálogo que se orienten a la construcción de categorías comunes, con tiempos y espacios específicos para estas tareas. Desde el inicio, debe incluirse a todos los actores involucrados, tanto de la academia como fuera de ésta; además, se debe trabajar de manera colaborativa en proyectos viables y dar acompañamiento puntual y continuo. Igualmente, es importante la difusión y la medición de los resultados. 
Cabe destacar que los enfoques inter y transdisciplinarios pueden enriquecer a la academia y generar una ciencia transformadora. De igual modo, pueden ser una guía o funcionar "como la estrella polar" (A. Polo, comunicación personal, diciembre de 2020) hacia la cual nos debemos encaminar. Todos los esfuerzos que se llevan a cabo en cada división son muy importantes, al igual que las propuestas que los expertos plantearon desde sus diversos ámbitos.

Finalmente, cabe aclarar que estas recomendaciones podrían aplicarse a otras instituciones de educación superior que compartan la importancia de adoptar la inter y la transdisciplina en los procesos de docencia y en la construcción colectiva del conocimiento; asimismo, deben reconocer la indispensable adecuación a los contextos particulares y la necesidad de resolver problemas complejos.

La buena respuesta, disposición y apertura de los divisionales, directores y coordinadores de cada programa académico, además de permitir la redacción de este artículo, nos muestra la necesidad de construir, en diálogo, el camino que la Ibero debe tomar hacia la inter y transdisciplina en los procesos de investigación, enseñanza y aprendizaje. Asumir estos enfoques puede favorecer la búsqueda de soluciones a los problemas actuales y abonar a la justicia social que buscamos: "dialogar con, caminar con, contribuir con otros en la consecución de mejores condiciones de vida desde la academia [...] desde la misión, la visión, la perspectiva, los planes, etcétera, que se ha propuesto la Ibero" (J. F. de la Fuente, comunicación personal, febrero de 2021). En suma, este artículo tiene como objetivo primordial hacer visibles los puntos fuertes de la Ibero rumbo a este camino.

\section{Agradecimientos}

A los directores divisionales: doctor Luis Javier Cuesta Hernández, director de la División de Humanidades y Comunicación (DHYC), maestro José Antonio Morfín Rojas, director de la División de Ciencia, Arte y Tecnología (DICAT), maestra Alma Polo Velázquez, directora de la División de Estudios Sociales (DES), doctora Marisol Silva Laya, directora de la División de Investigación y Posgrado (DIP), doctor Juan Fernando de la Fuente, director del Departamento de Reflexión Interdisciplinaria (DRI), doctora Mónica Chávez Aviña, coordinadora del Departamento de Reflexión Interdisciplinaria (DRI). A los directores y coordinadores de los programas académicos de la Universidad Iberoamericana Ciudad de México-Tijuana, por su tiempo, disposición, amabilidad y apertura para realizar este ejercicio. Agradecemos también a Adriana Argumedo, del Programa de Desarrollo Curricular (PDC), y a José Luis Gutiérrez Brézmes, integrante del Senado Universitario, por su valiosa orientación para la elaboración de este trabajo.

REFERENCIAS

Arce, M. Germán, D. et al. (1988). Capítulo II. La entrevista y el cuestionario. En E. de la Garza Toledo (Coord.), Hacia una metodología de la reconstrucción: fundamentos, crítica y alternativas a la metodología y técnicas de investigación social. México: UNAM.

Bernard, R. (2006). Research Methods in Anthropology: qualitative and quantitative approaches. Qualitative and Quantitative Approaches. Lanham: Altamira Press.

Carrizo, L., Espina Prieto, M. \& Klein, J. (2004). Transdisciplinariedad y complejidad en el análisis social. Unesco.

Castillo, M., Aragón García, M. \& Hernández Jaime, J. (2007). Los procesos de acreditación: desafíos para la Educación Superior en México. Revista Iberoamericana de Producción Académica y Gestión Educativa. Recuperado de https:// www.pag.org.mx/index.php/PAG/article/view/118/165
Constitución Política de los Estados Unidos Mexicanos [Const.]. Art. 3. ${ }^{\circ}$ Fr. VII. 11 de marzo de 2021 (México).

Decreto por el que se expide la Ley General de Educación Superior y se abroga la Ley para la Coordinación de la Educación Superior. Art. 7.․ 20 de abril de 2021.

Denzin, N., \& Lincoln, Y. (1994) Introduction: Entering the field of qualitative research. En E. G Guba y Lincoln Y. S. (Eds.), Handbook of Qualitative Research (M. Perrone, Trad.). California: Sage Publications.

Doctorado en Estudios Criticos de Género (DECG). Recuperado de https://posgrados.ibero.mx/sites/default/files/2017-11/ doctorado_estudios_criticos_genero_oct-17.pdf

Fam, D., Palmer, J., Riedy, C., \& Mitchell, C. (Eds.) (2017). Transdisciplinary Research and Practice for Sustainability Outcomes. Nueva York: Routledge.

104 - La caminos a la inter y transdisciplina de la Universidad Iberoamericana Celia Arteaga Conde, José Alberto Lara Pulido, José Alberto Gallardo Cruz, Carmina Isabel Jiménez Quiroga, Adriana Carolina Flores Díaz, Raúl Gutiérrez Patiño, Juan Manuel Núñez. DIDAC 78 (2021): 88-107 
Flores, F. \& Rubio, A. (Eds.). (2019). Género, transdisciplina e intervención social. México: UNAM.

Glaser, B. \& Strauss, A. (1967). The discovery of grounded theory. Nueva York: Routledge.

Guevara, A., Sanginés, A., Lara Pulido, J. A. \& Riojas Rodríguez, J. (2019). Investigación inter y transdisciplinaria en el cuidado de la Casa común: la propuesta del Centro Transdisciplinario Universitario para la Sustentabilidad (Centrus). En Fernández Anaya, A. González Jácome, G. Prado Garduño \& M. C. Torales Pacheco (Eds.), La Universidad Iberoamericana generadora de conocimiento. Un caleidoscopio. México: Universidad Iberoamericana.

Harding, S. (2002). ¿Existe un método de investigación feminista? En E. Bartra (Comp.), Debates en torno a una metodología feminista. México: PUEG-UAM-X.

Hernández J., Chumaceiro Hernández, A., Reyes Hernández, I. \& Argumedos de la Ossa, C. (2016). Universidad en América Latina, transdisciplina y redes de investigación colaborativas. Revista Cientifica. Teorías, Enfoques y Aplicaciones en las Ciencias Sociales, 8(18), 11-18.

Hernández Sampieri, R., Fernández Collado, C. \& Baptista Lucio, P. (1991). Metodología de la investigación. México: McGraw Hil.

John, B., Caniglia, G., Bellina, D. et al. (2017). The glocal curriculum. A practical guide to teaching and learning in an interconnected world. Lüneburg: Baden-Baden: [sic] Critical Aesthetics Publishing. Kelly, S. et al. (2016). Scoping the Potential for Interdisciplinary Postgraduate Programs at UTS, Australia: Institute for Sustainable Futures (UTS).

Kuhn, T. S. (2012). The Structure of Scientific Revolutions. Chicago: University of Chicago Press.

Maestría en Estudios sobre Migración. Recuperado de https:// posgrados.ibero.mx/maestriaenestudiossobremigracion

Mauser W., Klepper, G., Rice, M. et al. (2013). Transdisciplinary global change research: the co-creation of knowledge for sustainability. Current Opinion in Environmental Sustainability, 5, 420-431.

Merçon, J., Ayala-Orozco, B. \& Rosell, J. (Coords.). (2018). Experiencias de colaboración transdisciplinaria para la sustentabilidad. México: Copit-arXives Recuperado de
http://scifunam.fisica.unam.mx/mir/copit/SC0007ES/ SC0007ES.html

Morin, E. (1996). Por una reforma del pensamiento. Correo de la Unesco. Un sólo mundo, voces múltiples. Recuperado de https:// es.unesco.org/courier/fevrier-1996/reforma-del-pensamiento

Norgaard, R. (1999). Negotiating Expertise in Disciplinary "Contact Zones". Language and Learning Across the Disciplines, 3(2), 44- 63.

Rendón, P. (2021). Nuevos planes de estudio, una propuesta de la IBERO para hacer frente al futuro. Recuperado de https://ibero.mx/prensa/nuevos-planes-de-estudio-unapropuesta-de-la-ibero-para-hacer-frente-al-futuro

Sánchez Gómez, M. (2014). La dicotomía cualitativo-cuantitativo: posibilidades de integración y diseños mixtos. Campo abierto: Revista de educación, 1, 11-30.

Schäpke, N., Stelzer, F., Caniglia, G., \& Bergmann M. (2018). Jointly Experimenting for Transformation? Shaping. GAIA - Ecological Perspectives for Science and Society, 27(1), 85-96. doi: 10.14512 /gaia.27.S1.16

Universidad Iberoamericana (2018). Plan Estratégico Institucional. Rumbo 2030. Recuperado de http://rumbo2030.ibero.mx/

Universidad Iberoamericana (2020). Marco operativo para el diseño de planes de estudio. Recuperado de https://enlinea. uia.mx/plan_estudio/Marco_operativo_CDMX_2020.pdf

Vilar, S. (1997). La nueva racionalidad. Comprender la complejidad con métodos transdisciplinarios. Barcelona: Kairós.

Wanner, M., Hilder, A., Westerkowski, J. et al. (2018). Towards a Cyclical Concept of Real-World Laboratories. disP - The Planning Review, 54(2), 94-114. doi: 10.1080/02513625. 2018.1487651

Wiek, A., Withycombe, L. \& Redman, L. (2011). Key competencies in sustainability: a reference framework for academic program development. Sustainability Science, 6(2), 203-218.

Scholz, R. \& Steiner, G. (2015a). The real type and ideal type of transdisciplinary processes: part I- theoretical foundations. Sustainability Science, 10, 527-544.

Scholz, R. \& Steiner, G. (2015b). The real type and ideal type of transdisciplinary processes: part II- what constraints and obstacles do we meet in practice? Sustainability Science, 10, 653-671.

BIBLIOGRAFÍA RECOMENDADA

Cuesta, J. (2021). Libro blanco sobre el desarrollo de Programas Interdisciplinares en la Universidad Iberoamericana. Hacia la resolución de problemas a través de la Interdisciplina. Nuevas propuestas Multi/Inter/Transdisciplina. Debates y propuestas en la Universidad Iberoamericana. Documento de trabajo. Universidad Iberoamericana.

Leyva, X. \& Speed, S. (2008). Hacia la investigación descolonizada: nuestra experiencia de co-labor. En X. Leyva, B.
Burguete y S. Speed (Coords.), Gobernar (en) la diversidad: experiencias indigenas desde América Latina. Hacia la investigación de co-labor. Guatemala, Ecuador, México: CIESAS-FLACSO.

Wanner, M., Schmitt, M., Fischer, N. \& Bernert, F. (2020). Transformative Innovation Lab. Lünerburg, Berlín: Wuppertal Institut-Leuphana University of Lüneburg-Freie Universität Berlin.

\section{SEMBLANZAS}

Celia Arteaga Conde. Doctora y maestra en Antropología por el Centro de Investigaciones y

Estudios Superiores en Antropología Social (cIESAS), Ciudad de México; licenciada en

Educación Preescolar por la Secretaría de Educación Pública (sEP); licenciada en Estudios

La caminos a la inter y transdisciplina de la Universidad Iberoamericana $\bullet 105$

Celia Arteaga Conde, José Alberto Lara Pulido, José Alberto Gallardo Cruz, Carmina Isabel Jiménez Quiroga, Adriana Carolina Flores Díaz, Raúl Gutiérrez Patiño, Juan Manuel Núńez. DIDAC 78 (2021): 88-107 
Latinoamericanos por la Universidad Nacional Autónoma de México, Facultad de Filosofía y Letras (UNAM-FFYL). Académica de tiempo completo del Centro Transdisciplinar Universitario para la Sustentabilidad (Centrus), de la Universidad Iberoamericana, desde julio de 2020. Su formación académica se orienta a procesos sociales contemporáneos, con un enfoque crítico feminista y de género, que le ha servido como herramienta para el análisis e interpretación de eventos sociales, particularmente de temas medioambientales, de sustentabilidad y de fenómenos migratorios. Asimismo, se interesa por la educación en general y, particularmente, en la medioambiental, ya que la considera un pilar fundamental para el desarrollo social del país.

José Alberto Lara Pulido. Doctor y maestro en Economía por el Colegio de México (colmex) y licenciado en Administración por la Universidad La Salle. Actualmente es director del Centro

Transdisciplinar Universitario para la Sustentabilidad (Centrus), de la Universidad Iberoamericana. Es miembro del Sistema Nacional de Investigadores del CONACYT (nivel candidato). Ha sido consultor en temas de política pública y en materia social y ambiental, así como en modelación económica, para el Banco Mundial (вм), el Banco Interamericano de Desarrollo (BID), The Nature Conservancy, la Agencia de Cooperación Alemana en México (GIz), el Instituto Nacional de Ecología y Cambio Climático (INECC), el Fondo Mexicano para la Conservación de la Naturaleza (FMCN), ONU Medio Ambiente, el Consejo Nacional para la Evaluación de la Política Social de México (coneval) y el Fondo Mundial de la Naturaleza (WwF). Ha realizado publicaciones académicas en revistas arbitradas en temas de valoración económica de servicios ecosistémicos, mitigación y eficiencia energética, agua y pobreza, decisiones de transporte, comportamiento ambiental y expansión urbana.

José Alberto Gallardo Cruz. Biólogo y doctor en Ciencias por la Universidad Nacional Autónoma de México (UNAM). Académico de tiempo completo del Centro Transdisciplinar Universitario para la Sustentabilidad (Centrus). Estudia las relaciones recíprocas entre los atributos de las comunidades vegetales y las propiedades del paisaje. En particular, investiga la estimación de variables biofísicas y ecológicas a partir de la percepción remota; las metodologías para la estimación de atributos de la vegetación en grandes áreas y el análisis de la variación espacial de la diversidad; el efecto del cambio de escala en los procesos socioecológicos; el uso de tecnologías para la generación y el análisis de información 3D; la generación de modelos geoestadísticos de variables ambientales; y los procesos y las causas de la transformación de la vegetación (deforestación y degradación). Ha participado en proyectos para crear y fortalecer redes de colaboración interinstitucionales e interdisciplinarias (FOMIX, Laboratorios Nacionales y Problemas Nacionales).

Carmina Isabel Jiménez Quiroga. Licenciada en Economía por la Universidad Iberoamericana. Es asistente de investigación en el Centro Transdisciplinar Universitario para la Sustentabilidad (Centrus). Ha colaborado como asistente en diversos proyectos de investigación y consultoría. Su investigación se ha centrado en el desarrollo de modelos econométricos, el uso de Sistemas de Información Geográfica y en el análisis económico con enfoque de género y medio ambiente.

Adriana Carolina Flores Díaz. Académica de tiempo completo en el Centro Transdisciplinar Universitario para la Sustentabilidad (Centrus). Pertenece al Sistema Nacional de Investigadores del conacyt (candidata). Doctora en Ecología por el Instituto de Ecología, A. C., de Xalapa,

Veracruz. Es bióloga por la Facultad de Ciencias de la Universidad Nacional Autónoma de México (UNAM). Participa en redes colaborativas de investigación y vinculación

en temas de ecosistemas dulceacuícolas, ecología, cuencas y socioecosistemas, así como en el diseño del Observatorio Nacional de Sustentabilidad Socioecosistémica. Sus líneas

106 - La caminos a la inter y transdisciplina de la Universidad Iberoamericana Celia Arteaga Conde, José Alberto Lara Pulido, José Alberto Gallardo Cruz, Carmina Isabel Jiménez Quiroga, Adriana Carolina Flores Díaz, Raúl Gutiérrez Patiño, Juan Manuel Núñez. DIDAC 78 (2021): 88-107 
de investigación se enfocan en la dinámica socioecosistémica a diversas escalas y en los métodos transdisciplinarios para su análisis. Es cofundadora del programa Global Water Watch México. Colabora en el IPBES-ONU, donde participa en la constitución de la Red de Interesados (ONet) y, desde 2019, en el Panel Multidisciplinario de Expertos (MEP) de la Plataforma.

Raúl Gutiérrez Patiño. Doctor y maestro en Estado de Derecho y Buen Gobierno por la Universidad de Salamanca, España; maestro en Negociación y Relaciones Internacionales por la Escuela Jacobea de Posgrado, con estudios de titulación en desarrollo sostenible. Licenciado en Relaciones Internacionales por la Universidad Iberoamericana; y licenciado en Derecho por la Universidad Nacional Autónoma de México (UNAM). Ha participado como profesor invitado y conferencista en distintas universidades en Colombia, España y México. En 2018, fue reconocido como Jurista Emérito por el Comité Ejecutivo de la Abogacía Colombiana.

Actualmente es académico de tiempo completo en el Centro Transdisciplinar Universitario para la Sustentabilidad (Centrus). Su línea de investigación gira en torno al análisis del Estado de Derecho para la consolidación de la justicia ambiental y el reconocimiento de la personalidad jurídica de los recursos naturales.

Juan Manuel Núnéez. Doctor y maestro en Geomática con especialidad en Análisis Espacial y Percepción Remota por el Centro de investigación en Ciencias de Información Geoespacial (CentroGeo). Licenciado en Ingeniería Topográfica y Geodesia por la Facultad de Ingeniería de la Universidad Nacional Autónoma de México (UNAM). Investigador asociado del CentroGeo de 2006 a 2019. Actualmente se encuentra adscrito al Centro Transdisciplinar Universitario para la Sustentabilidad (Centrus). Es miembro del Comité Técnico Asesor de la Procuraduría Ambiental y de Ordenamiento Territorial (рАот), de la Ciudad de México, y del Sistema Nacional de Investigadores del conacyt desde enero de 2019. Ha participado en más de una docena de proyectos de vinculación sobre temas de monitoreo de recursos naturales y procesos urbanos con instituciones locales, y como investigador en proyectos de investigación en diferentes Fondos y Apoyos CONACyT.

\footnotetext{
${ }^{1}$ Los planes de estudio del Sistema Universitario Jesuita (suJ) corresponden a los programas académicos que fueron diseñados en 2012 y que están vigentes al momento de esta investigación. Esta precisión es importante, ya que en este 2021 han sido actualizados por los Planes Manresa, que se orientan hacia la inter y transdisciplina.

${ }^{2}$ Doctor Luis Javier Cuesta Hernández, director de la División de Humanidades y Comunicación (DHYc), maestro José Antonio Morfín Rojas, director de la División de Ciencia, Arte y Tecnología (DICAT), maestra Alma Polo Velázquez, directora de la División de Estudios Sociales (DEs), doctora Marisol Silva Laya, directora de la División de Investigación y Posgrado (DIP).

${ }^{3}$ Doctor Juan Fernando de la Fuente, director del Departamento de Reflexión Interdisciplinaria (DRI) y doctora Mónica Chávez Avińa, coordinadora del Departamento de Reflexión Interdisciplinaria (DRI). Históricamente, este departamento ha tenido la encomienda de promover y desarrollar estrategias para la formación integral de los universitarios, bajo una óptica de diálogo entre distintas profesiones. El ARU ha sido un espacio de reunión para los estudiantes de todas las carreras y para fomentar el pensamiento crítico, a partir del análisis de temas transversales y socialmente pertinentes.

${ }^{4}$ El objetivo de contar con esta estampa etnográfica es registrar la conversación y ubicar el momento en el que se llevó a cabo cada entrevista.

${ }^{5} \mathrm{El}$ instrumento está disponible en https://forms.gle/zWB6Sj8FYtUzVCRn8

${ }^{6}$ Consulte el listado de los programas en el Anexo 2, disponible en: https://docs.google.com/document/d/1gm2ikWDzYCYKdZ7hPx-R8tHwMIobukFjQotvlonyQrM/edit?usp=sharing)

${ }^{7}$ Hubo programas que recibieron más de una respuesta: coordinador de programa, director de departamento o académicos que participan en coordinación o ejecución del programa.

${ }^{8}$ Los programas se agruparon de acuerdo con la rama del conocimiento a la que pertenecen, ya que la estructura administrativa de la Ibero no permitía hacer un análisis por tipo de ciencia o disciplina acerca de la percepción de la inter y transdisciplina en la universidad. Los programas de cada grupo se pueden consultar en el Anexo 2.

${ }^{9}$ Constitución Política de los Estados Unidos Mexicanos y Ley General de la Educación Superior.

${ }^{10}$ Libro blanco sobre el desarrollo de Programas Interdisciplinares en la Universidad Iberoamericana. Hacia la resolución de problemas a través de la interdisciplina. Nuevas propuestas. Multi/Inter/Transdisciplina. Debates y propuestas en la Universidad Iberoamericana.

${ }^{11}$ El objetivo de este seminario es reflexionar sobre el bien común para promover actividades académicas que favorezcan el compromiso social por parte de los miembros de la comunidad de la Ibero, así como de personas y organizaciones que busquen este mismo propósito. Información disponible en https://biencomun.ibero.mx/fundamentacion

${ }^{12}$ Véase https://genero.ibero.mx/

${ }^{13}$ Véase https://sustentabilidad.ibero.mx/
} 